\title{
Disparities between Explanatory Models of Health Clients, Healthcare Providers and Health Insurer
}

\author{
Christine J. Fenenga ${ }^{1}$, Robert Kaba Alhassan ${ }^{2}$, Stephen Duku ${ }^{3}$, Wendy Janssens ${ }^{4}$, Daniel Arhinful ${ }^{5}$ and Inge \\ Hutter $^{6}$ \\ 1. Population Research Centre, Department of Demography, Faculty of Spatial Sciences, University of Groningen and at the \\ Amsterdam Institute for Global Health and Development, Amsterdam, The Netherlands \\ 2. Noguchi Memorial Institute for Medical Research, University of Ghana and the University of Amsterdam, The Netherlands \\ 3. Noguchi Memorial Institute for Medical Research, University of Ghana and the Free University of Amsterdam, The Netherlands \\ 4. Free University of Amsterdam, The Netherlands \\ 5. Noguchi Memorial Institute for Medical Research, University of Ghana, Accra, Ghana \\ 6. International Institute for Social Studies, The Hague, The Netherlands
}

\begin{abstract}
We present qualitative data from a study in Ghana (2011), where the National Health Insurance Scheme (NHIS) was introduced to improve access to health care. In 2011 membership enrolment and retention in the scheme was stalling. To obtain better insights into socio-cultural factors that influence utilization of healthcare services and the NHIS this study compared Explanatory Models of healthcare clients with those of primary healthcare providers and the NHIS regarding illness, the need for, the quality of, and the control over healthcare and health insurance services. We found critical disparities in socio-cultural beliefs and perceptions of healthcare and health insurance between these three stakeholder groups, such as the clients' holistic view on illness versus healthcare providers' bio-medical view; the clients' inter-relational focus in perceiving quality of services versus the providers' medical technical focus. These differences are leading to misconceptions, blame practice, poor services, non-adherence and low trust. The findings increase our understanding of clients' behavior and that of their service providers. We conclude with key messages for policy leaders and operational managers that can guide them in improving services and facilitating client trust and interest to participate in health insurance and utilize healthcare services.
\end{abstract}

Key words: Explanatory Models, communication, trust, health insurance, Ghana.

\section{Background}

In many low-income countries health insurance is being introduced to increase access to healthcare, in particular for the vulnerable and poor population $[1,2]$. This is expected to provide poor households with risk protection. In countries with unfavorable health indicators, such as high maternal and infant mortality rates, health insurance has been instrumental to improve this situation [3-5]. The challenges in reaching out to the full population have been demonstrated by different studies $[6,7]$. A wide range

Corresponding author: Christine J. Fenenga, MPH, Ph.D., research field: socio-cultural determinants of health. of barriers have been found to influence enrollment in health insurance, such as cost, distance to the facility, quality of care, and timing of premium payment [8-13]. It is also believed that socio-cultural factors such as local beliefs and social norms play a significant role in access to care and medical adherence [14, 15].

Our study context is Ghana, where the government introduced a National Health Insurance Scheme

(NHIS) in 2003. This mandatory scheme was to replace the 'fee for service' system and ensure better access to quality healthcare for all citizens in Ghana. Considerable progress has been made and in 2013, at its ten-year anniversary, $34 \%$ of the population (over 
8.5 million people) was actively using the scheme while still a large segment of Ghana's population did not benefit from the scheme. Several studies identified factors, such as low education, financial barriers or poor quality of services, as responsible for this low enrollment coverage [2]. Individuals from poorer households were less likely to enroll compared to those from rich households despite the core poor, pregnant women, pensioners and people below 18 years and above the age of 70 being exempted from paying premium [16].

In 2011 the National Health Insurance Authority (NHIA) was keen to obtain a better insight in community members' perceived socio-cultural barriers that influence participation in the scheme and requested for this study. This paper there for explicitly studies how motivation to utilize services is embedded in people's socio-cultural beliefs, values, expectations, roles and relationships. Literature review revealed few studies looking at the intersection between patients and medical care providers [15, 17]. Effective provider communication is mentioned as a key problem and believed to lead to risk in non-adherence to treatment and low trust. One study looked at perceptions and experiences of healthcare providers and clients in a health insurance setting, with specific attention to behavior of healthcare providers. We believe that to obtain a comprehensive understanding of clients' motivation to utilize services, needs looking at three angels of the client-provider-insurer tripod (Fig. 1).

While we focused on clients ${ }^{1}$ (in their community) and their interface with the healthcare providers and NHIS, we also explored the interface between NHIS and healthcare providers, as we expect that disparities between these two stakeholders can potentially negatively influence behavior of client. Findings are to support the NHIA to further improve their policy and practice.

\footnotetext{
${ }^{1}$ The word clients in this manuscript means clients and potential clients of the NHIS.
}

\section{Explanatory Models and the Link to Communication}

To design our study and analyze and interpret the data, we adopted Kleinmans' socio-anthropological model on health systems [18, 19]. His theory is specifically relevant for studying the interface between the different stakeholders in the health system. Since its' inception the NHIS has steadily campaigned, resulting in a high community awareness and acceptance about the program, hence we found the Diffusion of Innovation Theory less suitable for this study. Kleinman [18] introduced the socio-anthropological model to explain clients' perceptions of illness and choice of care through the definition of three healthcare systems: the popular (or domestic), folk (or traditional) and professional (Western). Figure 2 presents this model. These healthcare systems are organized by sub-systems of socially legitimate beliefs, values, expectations, roles and relationships, called clinical realities constructed by Explanatory Models (EM's).

EMs differ across societies and between individuals. People base their EMs on their acquired insights and knowledge obtained during day-to-day life, professional training and instruction and protocols at their work place. A doctor's concept of illness is found to relate to the bio-medical definition of disease, determining this as a problem of the organ itself $[20,21]$. While patients may recognize certain terms, such as malaria, they have limited bio-medical knowledge of the disease and its' etiology [22]. The term illness in the patient's EM is a more subjective response of being unwell, of suffering and the perceived need and expectations of treatment [20]. This will be different for an insurance officer, whose perceptions on insights and knowledge is biased from a health-economic and business training. Healthcare relationships can be seen as transactions between different explanatory models. Inability to decode each other's verbal and non-verbal language may easily cause conversational asymmetry that can lead to mistakes in service provision and reduce reciprocal trust as revealed in various studies [17, 23, 24]. 


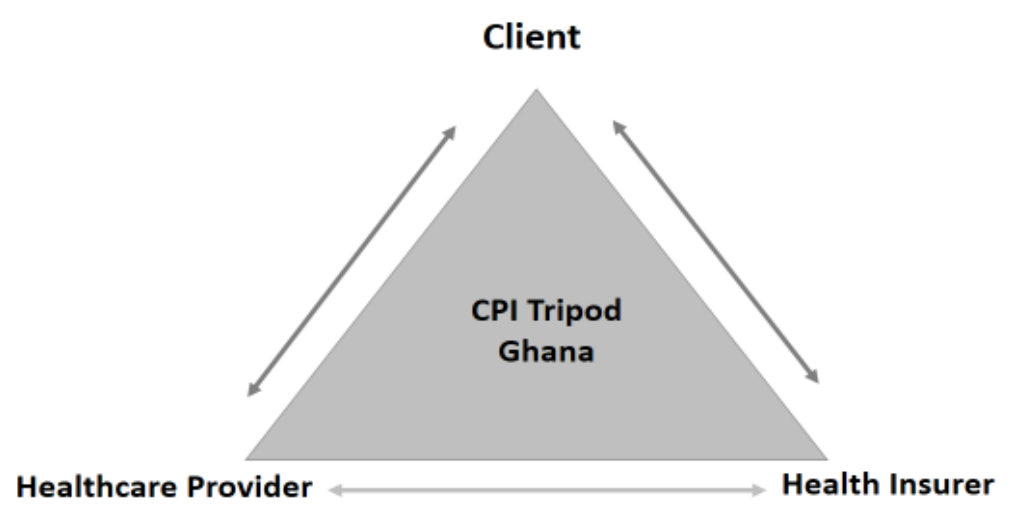

Fig. 1 Client-Provider-Insurance tripod.

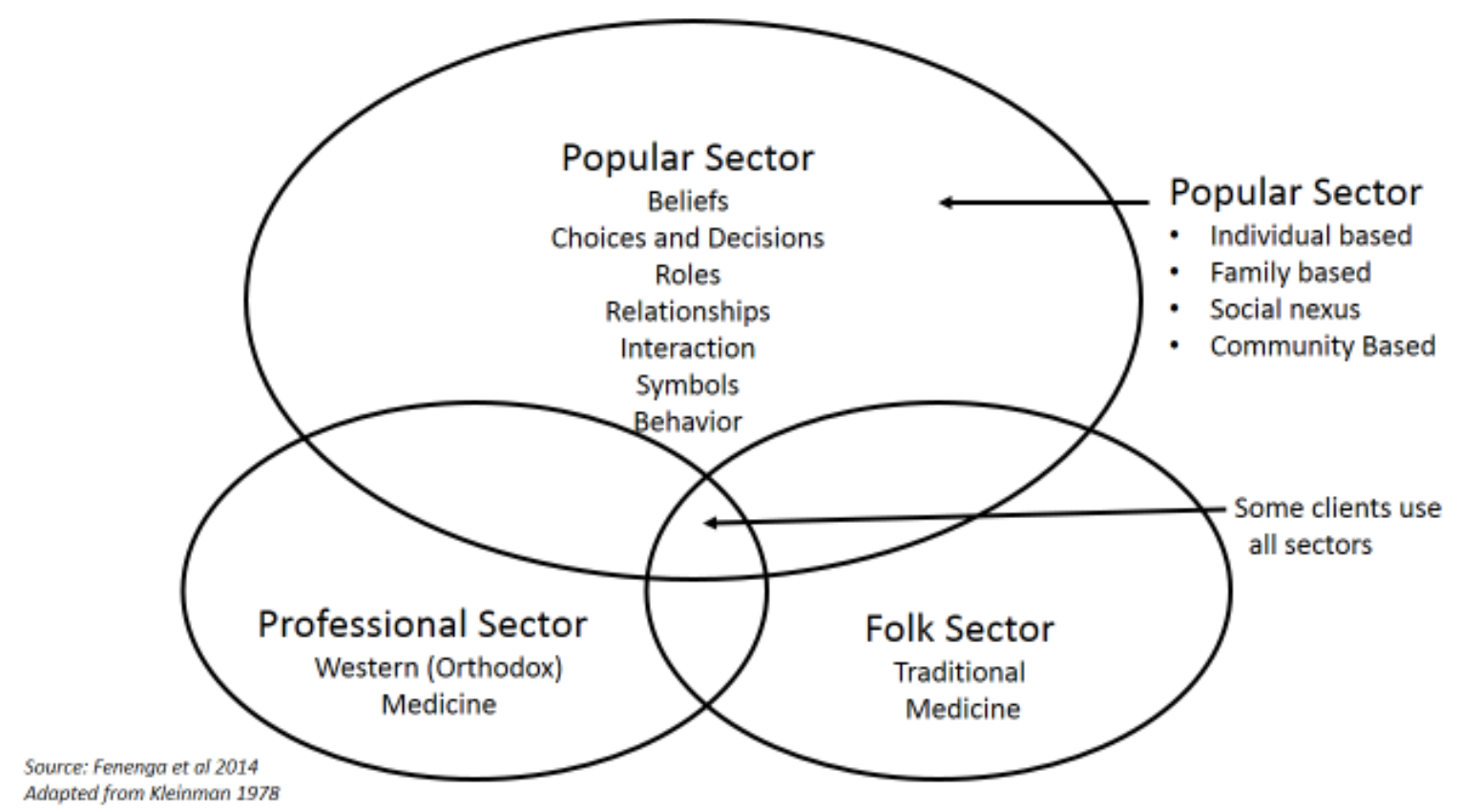

Fig. 2 Healthcare systems according Kleinman (source Fenenga et al 2014).

Recognizing differences between EMs helps an understanding of how people make decisions. If a health worker elicits the client's EM, he acquires insight in the beliefs the client holds about his illness, the personal and social meaning he attaches to his illness, and the expectations about what will happen. This insight is expected to facilitate bridging the gap between EMs and to build trust [19]. Besides differences in EMs, healthcare relationships are also featured by differences in power (such as social class, professional knowledge, ethnicity, gender) and trade-offs (what do I get out of this interaction). This power inequality may cause another challenge in the interaction.

\section{Method and Data Management}

Our study was conducted in 2011 in two administrative and socio-demographically distinct regions in Ghana: Greater Accra and the Western Regions. To obtain an in-depth understanding of the perceptions of clients (insured and not-insured), healthcare providers (public and private) and health insurance staff, a participatory action approach (PAA) was employed, using a range of interviewing techniques 
to elicit the views and opinions of participants and to allow for triangulation and comparison of data (Table 1). The PAA was chosen for its specific features of awareness raising, exchange of views and mutual learning among participants [25]. Our focus of the study was predominantly on the perceptions of the users of the NHIS, hence most interviews were conducted with clients.

We recorded perspectives of clients (older than 18 years of age) in their own communities in both study regions. Locations were defined with support of the district authorities. Interviews were conducted in local languages, using predefined, translated topic guides for which support was obtained from the linguistic department of the University of Ghana.

Providers' perspectives were collected from nurses, medical assistants, pharmacists, lab technicians and midwives and few District Directors of Health Services at their work-place. The views of the health insurer involved interviews with district scheme officers, claim officers, public relation officers, regional managers, monitoring and evaluation officers and some departmental directors at national level.

Average duration of individual interviews was 45 min, while FGD took averagely $90 \mathrm{~min}$. For the three stakeholder groups a pre-tested topic guide was defined based on Kleinman's theory of EMs and common inductive themes that arose during interviews (Table 2).

All interviews were recorded and transcribed verbatim to reduce errors. Field notes were also taken as complementary information. The interviews were conducted by the research assistants and supervised by the investigators. Data was coded, categorized according to identified themes and analyzed using $\mathrm{N}$-Vivo 9 to interpret their meaning and form well-founded conclusions. The analysis was conducted by a team, and three investigators checked each-others findings. When two people disagreed, the third person did the final check.

Findings were validated and triangulated through two workshops organized for purposively selected clients, healthcare providers and health insurance staff in both study regions. Purposively selected stakeholders (averagely 20 people per stakeholder group in each region) were invited to the regional capital and were presented the results by stakeholder group. Findings were shared in different formats: through oral presentation, exhibited pictures and quotes (anonymized) followed by feed-back and discussions. No modification of results was required. Subsequently, stakeholder groups presented their own results to the

Table 1 Interviews with stakeholders.

\begin{tabular}{|l|l|l|l|}
\hline Stakeholder groups & Interview techniques & Language & Location \\
\hline $\begin{array}{l}\text { Clients (insured and not-insured, } \begin{array}{l}\text { all above 18 years of age) } \\
\mathrm{N}=223\end{array} \\
\text { 22 Focus Group Discussions (average 9 } \\
\text { participants; mixed compositions } \\
\text { male/female, insured/not insured) } \\
\text { 20 Individual Health Histories } \\
\text { 3 Key Informant Interviews }\end{array}$ & $\begin{array}{l}\text { Local languages: Akan, } \\
\text { Dangme, Nzema } \\
\text { people's home; } \\
\text { FGD at public } \\
\text { community building }\end{array}$ \\
\hline $\begin{array}{l}\text { Primary Health Care Providers } \\
\text { public and private) } \\
\mathrm{N}=22\end{array}$ & $\begin{array}{l}\text { 22 In-depth Interviews } \\
\text { (including 4 officers at regional level) }\end{array}$ & English & $\begin{array}{l}\text { Primary Care Facilities, } \\
\text { Offices }\end{array}$ \\
\hline $\begin{array}{l}\text { Health Insurer (district, regional, } \\
\text { national level) } \\
\mathrm{N}=16\end{array}$ & $\begin{array}{l}\text { 16 In-depth Interviews } \\
\text { (including 4 officers at national level) }\end{array}$ & English & $\begin{array}{l}\text { Offices (district, } \\
\text { regional, national) }\end{array}$ \\
\hline
\end{tabular}

Table 2 Common topics in interview guides of clients, healthcare providers and health insurance.

\begin{tabular}{|l|l|}
\hline Common topics & Probing terms in the interviews \\
\hline Illness and disease & Meaning, causality, severity \\
\hline Need for healthcare and health insurance services & Cultural beliefs, awareness, value, severity, financial ability, efficacy \\
\hline Quality of services (healthcare/health insurance) & Definition, expectation, experience, sustainability \\
\hline Respective roles & Traditions, expectations, level of influence, power relations \\
\hline
\end{tabular}


other two stakeholder groups, allowing sensitization and mutual learning, while identifying similarities and differences in Ems relating to illness, need for, quality of, and control over healthcare and health insurance services. Discussions were facilitated by the investigators and revealed that interaction between stakeholders in Western Region was not as open as in Greater Accra. Clients were more reluctant in voicing their opinions in presence of healthcare providers and health insurer. Based on the discussion, suggestions for solutions to overcome the disparities raised were sought from the participants. These were incorporated in the design of an intervention.

\section{Results}

We present the EMs of respective stakeholders on illness, need for, quality of care and control over healthcare and health insurance services (Table 2).

\subsection{Perspectives of Healthcare Client}

\subsubsection{Illness}

In Ghana the popular-natural perspective of the clients features different worlds of causation: the individual (that matches with biomedical model); the natural (excessive cold, heat, pollution); the social (witchcraft), the supernatural world (gods, spirits or ancestors), or a combination of these. Their logic, the way they look at illness, shows a variety as quoted by a woman in Western Region: 'You see, diseases can be grouped into two, the physical and the spiritual. So if you go to hospital and it [ref. the illness] does not stop, it means it is spiritual so you should go to prayer camp' (female, GAR). '... There are some illnesses that are said to be caused by harm afflicted on you spiritually....you have to remove the harm or curse before doing something else' (female WR). This shows that perceived causality of illness determines to a large extent the drive and place to seek care. Drive and place can also be led by experienced physiological factors: 'For me, I am pregnant. If anything happens to me, my mother insisted, I should go to the hospital' (female WR). In both regions we found many clients mentioning the influence of close relatives on how they deal with their health problem. It shows the way close relatives and friends believe and advice on treating illness and seeking care is important.

\subsubsection{Need for Healthcare and Health Insurance}

First 'emergency' care traditionally is sought from home. 'When ...someone is sick, I first break leaves, cook it and drink it.... If the sickness is not getting better, then you bring it [the sickness] to the hospital' (female WR). Religion is found fundamental in many Ghanaian EMs. Seeking a cure to relieve suffering and removing the harm or curse through intense prayer sometimes sought from prayer camps is common practice. Also, practical circumstances such as poor financial situation could determine the drive for seeking healthcare: 'When I have no money and I am sick, I go to the drug-shop. I can tell him that I have three Ghana Cedis. He would look for drugs of low cost. So I decide the cost that I can afford' (male $G A R)$. Others prefer visiting the professionally trained doctor, sometimes influenced by personal relationships: 'I know the doctor. He was with me at senior school so......I know he will take care of me well. So relationship also counts' (female GAR).

Responses demonstrated that everyone knows the NHIS and has some level of understanding of the concept. 'An illness can occur to you any time. With the insurance you have already paid so when I fall ill I can go to hospital and access care. That motivated me to do it' (female WR). People appreciate NHIS's effort to meet their needs. 'Last time someone came to talk extensively about insurance. They made it clear that even at the local drug shop they made agreements so that if the drug is covered.., people can be served here' (female GAR). We also found some clients had strong feelings about traditional care currently falling outside the scope of the insurance package. 'All we are saying is that he [herbalist] is someone who has been helping us a lot here. I will appreciate if they could give him 'wooloko' [referring to certificatellicense] so that he 
can look after us' (female GAR). In both regions we found that clients experience barriers to enroll in the scheme due to slow administrative procedures (enrollment/renewal) and perceived poor quality of the benefit package at the health facility.

4.1.3 Quality of Healthcare and Health Insurance

Clients' perception of quality healthcare shows the strong connection associated with comforting the ill body and mind. 'When the illness is serious, like my illness ...... I would like a nurse around to hold me, put me on a bed and ask me exactly what's wrong with me and knows what drugs to give me. That is what I expect' (female WR). Generally, clients refer more to inter-relational aspects of quality (respect, responsiveness) than to the biomedical aspect. 'At times you are there, the nurses don't pay any attention to you. They don't bother. That is one issue that worries us a lot' (male GAR). For some, quality also relates to availability of adequately trained staff, diagnostics and quick services. Our data reveals that people typically find that this is offered by private healthcare providers, a reason for many clients to prefer private clinics above public. Perceptions related to quality health insurance services show the importance of an easy registration and renewal process, accessible inquiry facilities and a comprehensive insurance package. 'Why I realized that it was not good?...most of my siblings and friends that have insurance looked disappointed' (Male GAR). This shows that opinions and experience of close relatives and friends influence people's opinion and behavior.

4.1.4 Control over Healthcare and Health Insurance Services

When asking clients how they position themselves when visiting the doctor, they explained that they perceive their position as 'receiver' of care, 'undergoing' the services, while that of the doctor is making decisions and providing the care. Most clients feel uncomfortable asking questions, although they are conscious about their rights as a patient. They mention being reluctant filing complaints at clinics, fearing repercussions. Other say it will not change the services anyway. This leads to cases of helplessness, not knowing where to file their complaints: 'Where can we go? To the police?' (Male WR). They observe considerable differences in how facility heads manage their operations and handle patients: 'As for the private clinics, supervision is good and very intense, more than at government.' A common complaint concerns the differences in treatments between the non-insured and the insured, with poor treatment for insured. Clients stress that 'government' and NHIS should keep their promise to provide quality services to all people. They requested the NHIA to communicate transparent information and monitor providers to ascertain non-discriminating services for insured and non-insured.

\subsection{Perspectives of the Healthcare Provider}

\subsubsection{Illness versus Disease}

Healthcare provider perspectives on disease are largely informed by their professional medical training in the 'germ theory' where there is always a 'cause-effect' principle. Offering services to someone with a health problem means finding and curing a biological cause such that the person recovers quickly. 'We are to follow due processes....to ensure patients get well without medical complications, by treating a particular infection.....' (Nurse, private hospital GAR). This notion is directly associated with the providers' logic and handling of clients in the service delivery process [26]. '.....it encompasses the availability of right equipment, functional [medical] equipment...... and our responsiveness to emergencies' (Deputy Director of Health, GAR). The same interviewee remarked that the social and psychological aspects of the reporting case often receive minimal attention if any at all.

4.2.2 Need for (professional) Healthcare and Health Insurance

Providers stressed the importance of clients reporting without delay, to allow early diagnoses and 
treatment. Data revealed a general 'blame game' where healthcare providers attribute undesired treatment outcomes to late reporting and non-adherence to treatment regimen and review dates. A pharmacist in GAR explained 'these people [clients] will never come on time.....they first go in cycles to prayer camps.........before coming to hospital in bad state'. Asked why providers think clients adopt this health seeking behavior, none of the health professionals identified a possible lapse on their side. The problem was mainly seen to be a 'bad behavior of clients' as mentioned by a nurse in WR.

On the need for health insurance, majority of the health staff acknowledged the importance of health insurance to clients: '....the NHIS in particular has come to help the poor people.....especially those who cannot afford' (Lab technician GAR). A deputy director of pharmaceutical services in GAR indicated '...it improves accessibility and choice by the client....it makes people have value for their money......' A few health workers had some reservations on the benefits of the NHIS but added there is no other option since 'it is a government policy (Nurse Assistant in WR). A nurse in GAR remarked '...the NHIS is helping the patients and not the hospitals......due to late payment of claims by the insurance people.....'

4.2.3 Perceived Quality of Healthcare Services and Health Insurance Services

Healthcare providers' perception of healthcare quality seems predominantly influenced by medical technical ascriptions of what constitutes quality. A deputy director of pharmaceutical services in GAR indicated that 'quality encompasses quality medicine, good storage of medicine and quality dispensing....' A deputy director of nursing services in GAR stated that 'quality healthcare entails providing services that are aimed at achieving our goal...abiding by the protocol or the guidelines that are available'. Responses from health staff at national, regional and health facility levels consistently emphasized medical-technical dimensions over inter-relational components of quality care and indicating that health facilities were performing well in these technical areas. Only a few healthcare professionals understood quality care to include tailor-made services that aim to satisfy the patient, as a health administrator in GAR said: 'quality care includes the attitude of staff towards clients'. He admitted his hospital (like other health facilities) has not paid much attention to this, largely due to limited or no active participation of clients and community groups in quality improvement efforts.

4.2.4 Control over Healthcare and Health Insurance

Healthcare providers (especially clinical staff) perceive their role in the service delivery process mainly as trained professionals responsible for disease management. Those in private facilities consider satisfying patients to attract better patronage as another key responsibility. 'When the clients are happy they [clients] will always come.......and this helps the clinic...' (Nurse in Greater Accra). A few of the providers also mentioned patient education on rights and responsibilities as their role in contributing to quality healthcare delivery. Health providers' role in health insurance promotion came up in only two cases during an interview with one director in the Western region and another in Greater Accra, where they mentioned unprofessional practices such as false claims, provider-induced demand, and avoidance of poly-pharmacy as critical areas in which providers could help sustain the NHIS. Health professionals also indicated that because of the generous nature of the NHIS, (without co-payment for all visits and services) the majority of clients take advantage of and abuse the system. '........some patients even attend the clinic and take medicines and give to their relatives and friends who are not insured....' (Lab Technician in GAR). A pharmacist also bemoaned the way patients frivolously visit the out-patient department because they possess an insurance card. 'Patients just come to hospital with the least thing..... a small headache.....' 


\subsection{Perspectives of the Health Insurer (NHIS)}

\subsubsection{Illness versus Disease}

The NHIS objective states 'this initiative by the government is to secure financial risk protection against the costs of healthcare services for all residents in Ghana' [27]. When talking with the NHIS staff they perceive disease as a financial risk for clients losing income. In order to reduce (catastrophic) health expenditures the disease needs to be cured effectively. Responses were emphasizing predominantly managerial issues, such as 'reducing financial costs through rapid response with the adequate quality treatment'. Although interviewed health insurer staff all use the biomedical definition of disease (the NHIS benefits package covers $95 \%$ of diseases, based on the bio-medical diagnosis and treatment), some recognize the notion of social cultural beliefs and values used by the clients. While traditional care (offered by spiritual healers; herbalists, fetish priest) is not part of the NHIS package, some discussions are ongoing on possible inclusion of some aspects of traditional care in health insurance benefit package.

4.3.2 Need for Healthcare and Health Insurance Services

NHIS staff believes that once an individual falls ill the need to access quality health care is crucial. The scheme is perceived as important, in particular for the poor and vulnerable. Early and effective treatment will control the costs of healthcare and prevent clients' loss of income.

4.3.3 Quality of Healthcare Services and Health Insurance Services

The National Health Insurance Authority perceives the quality of healthcare services to clients from both medical-technical and interpersonal perspectives. They expressed the determinants of healthcare quality as appropriate diagnostic processes and procedures, dispensing of quality drugs, fair queuing system, short waiting times, friendly attitude of staff and accurate and timely claims processing. However, emphasis was placed on the technical quality of care through effective diagnosis and treatment since in their view the outcome of the treatment is paramount. They indicated that the healthcare providers are therefore monitored against these technical quality determinants: 'The right caliber of people should be there to deliver service. They should be able to provide both clinical and diagnostic services where necessary. The issue of waiting period should be reduced so that clients will not waste too much time at the facility. There should also be right attitude of staff to clients' (Scheme Manager, WR).

They perceive the quality of NHIS services to be based entirely on managerial, technical criteria. They define the determinants of NHIS quality services as the ease of registration and registration time, waiting period to acquire NHIS card and the availability of information on the importance and convenience of the NHIS at the time of registration. 'When we talk of the quality of services, one is the time, that is the period of time the person spends when he comes to register, and then the promptness with which we are able to address the concerns of the person' (monitoring \& evaluation officer GAR). They also mentioned information dissemination on the NHIS benefits package, drugs list and accredited facilities and pharmacies in the catchment areas of registered clients as a core component of their mandate.

\subsubsection{Control over Healthcare and Health Insurance}

The NHIA believes that their role in health service delivery in relation to healthcare providers lies in the area of monitoring health providers' quality of services, providing feedback on this and prompts payment of claims to providers. 'We go into their services such as right machines or equipment, labs, sanitation is good, how they receive patients and whether their waiting area is quality' (Regional Manager, WR). They inspect, assess, approve and accredit healthcare providers through the application of an accreditation tool to provide healthcare services to clients of the NHIS. In 2011 for example, 3,828 healthcare providers were accredited to provide 
healthcare services under the NHIS. They further indicated that their role in health insurance uptake involves ensuring that the waiting period of thirty min for registration and of three months for acquiring an NHIS ID card is reduced to the barest minimum in most districts. However, they did admit some areas of concern that need improvement were inadequate information on the NHIS benefits package at the registration point, long waiting periods of over four months to receive ID cards in some districts and poor attitudes of some staff towards clients.

The NHIS staff also held the view that healthcare providers and clients have equally important roles to play to ensure the effective and efficient functioning of the health insurance scheme: Healthcare providers have to ensure that NHIS card bearers are provided with the right information about the benefits package, treated and given the appropriate drugs on the NHIS drugs list, without having to make any co-payments for the treatment. They also expect healthcare providers to fill in their claims forms appropriately and file their claims on time to avoid any delays in the reimbursements. Clients' role is to show interest in the NHIS by enrolling and renewing their membership in the NHIS on time, encouraging their friends and family members to also enroll. They also mentioned that abiding by the gate-keeping system is an important role that clients have to play to ensure that they get the greatest benefit out of the schemes, since jumping the gate-keeping system to higher level facilities will mean clients will have to pay out of pocket for their treatment even though they are enrolled.

\section{Comparison and Discussion of EMs and Policy Recommendations}

Earlier studies of patients, pointed at the importance of effective communication between patients and medical staff to bridge differences in perspectives, leading to a chain of events such as patient trust and adherence [14, 17, 23]. In the setting of Ghana, described in this paper, perceptions of three closely interrelated stakeholders are explored: clients, healthcare providers and NHIS. Our study aimed to identify critical discrepancies that influence trust, utilization of healthcare and enrolment in Ghana's NHIS. We compared their perceptions on illness, need for quality of and control over healthcare and health insurance services (Fig. 3).

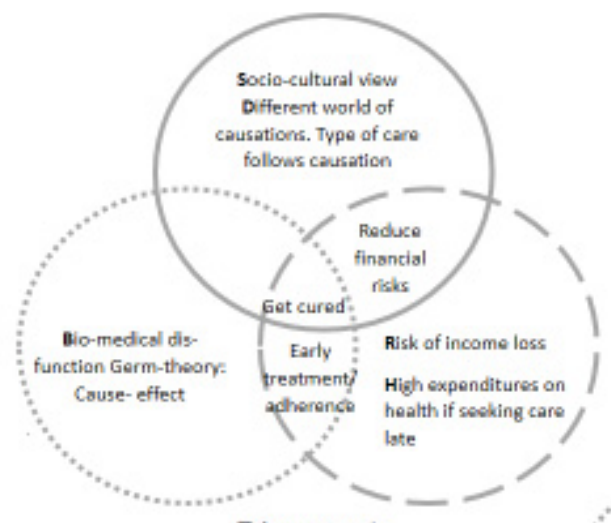

Diagram 1:

Illness-Need for service

Client:

Healthcare provider: ...............

Health insurer:

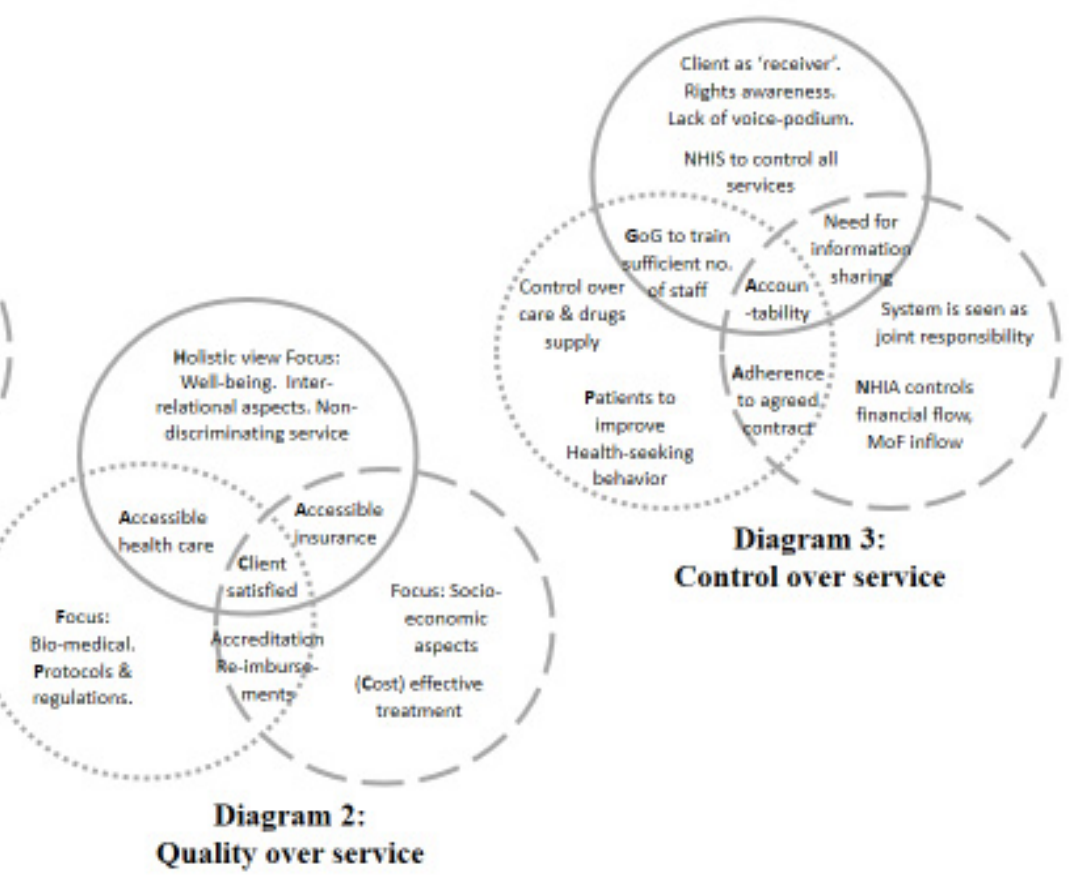

Fig. 3 EM comparisons. 
In our analyses, we found people's view on 'need for services' logically following their perceptions on 'illness/disease'. Hence 'illness' and 'need for services' is combined in one diagram.

Diagram 1. Clients use their world view to understand their sickness, often conceptualizing it on the basis of non-physiological factors. In contrast, healthcare providers and NHIS predominantly use the biomedical model to explain disease. We found clients not being well understood and therefore being reluctant to dialogue with healthcare providers. This results from practicing 'different cultural language' in combination with information asymmetry and power difference. Clients fear repercussions if they comment on treatment and are unhappy with the little information they receive about the diagnosis, the prescription and use of drugs. Asymmetric information can explain the persistent dependency position and limited influence (power) of clients in the tripod. On the other hand, the healthcare provider faces negative consequences from poor knowledge on clients' beliefs and values. They misunderstand and misinterpret client behavior such as late attendance or non-adherence to treatment. Clients, again, sometimes experience difficulties in understanding why the NHIS does not cover treatment of traditional practitioners as these often relate more to their own belief of illness and curing. The NHIS, adhering generally a biomedical definition, seems more open and aware of clients' cultural values and perceptions. The socio-economic view of the health insurer is a better match for the client's EM. Reducing the risks of catastrophic health expenditure is a major concern for many of the clients' responses.

Policy recommendations:

- Health professional training curricula should institutionalize training modules on socio-cultural beliefs of healthcare clients and social communication skills to help address the client-staff perception gaps.

Healthcare and health insurance professionals should enhance information sharing and offer understandable instructions to clients, consonant with the client's language (through using a variety of communication modes such as brochures, videos, text messages) to reduce the information asymmetry and power imbalance.

- The NHIS should continue exploring inclusion of some aspects of traditional health services, demonstrating their interest beyond the orthodox (western) health sector.

Diagram 2. In the analyses of EMs on quality of services we identified an entrenched position by healthcare and health insurance providers, emphasizing medical-technical quality and centered on the availability of good equipment and medicines and adherence to protocols as more important than clients' perceived quality of care. Recognition of inter-relational quality aspects as part of the client's holistic view will influence the experience, trust and satisfaction of clients in particular, which is important for enhancing utilization and enrollment. Another divergence relates to non-discriminating services (i.e., prescription of generic versus brand drugs, unexplained differentiation in waiting queues for insured and non-insured). Without providing adequate education to clients (and providers) on the quality of generic drugs, insurance clients may feel misled by the NHIS information. On the other hand, frivolous utilization of services or over-consumption (or over-prescription) of drugs is perceived by providers and NHIS as undermining quality care.

Policy recommendation:

- Healthcare providers and NHIS should engage clients and/or organized community groups/associations in their routine monitoring and feed-back on staff performance to make health staff more accountable to clients and promote client-centered quality care delivery.

- Health Professional Regulatory agencies (i.e. Medical and Dental Council, Nurses and Midwives Council) should include client care and non-technical quality care dimensions in their professional licensing and certification processes. 
- Regulatory agencies and Ministry of Health should effectively collaborate in post accreditation and credential monitoring using a common tool encompassing perceived and technical quality indicators.

Diagram 3. Control of services shows fewer divergences in the three EMs, though trust in the scheme is compromised by imbalanced power positions and by management and implementation challenges (e.g. slow processing of ID cards, delays in reimbursements). Clients expect healthcare services to be good and being supervised and monitored by the NHIS. This implies a rather passive role for clients themselves, which healthcare providers and NHIS likes to see changed. The traditional power between clients and healthcare providers changed with the introduction of the insurance scheme as a third actor with considerable (financial) power. This could explain the critical remarks by healthcare providers regarding NHIS reimbursement tariffs and delays in payment. According to healthcare providers they can guarantee correct services to clients on condition of timely reimbursements of claims by the NHIS. These management challenges are according to the NHIS partly caused by the healthcare providers (e.g. incorrect claims, late submission). The NHIS inspection role demonstrated that medical technical quality of many facilities does not meet the accreditation criteria. These standards however exclude perceived quality criteria.

Policy recommendation:

- Healthcare providers and NHIS should engage clients and/or organized community groups/associations in their routine monitoring and feed-back on staff performance to encourage a more active role for clients (empowerment) and enhance communication. In addition this routine monitoring will make health /insurance staff more accountable to clients and promote client-centered quality care delivery.

- Healthcare providers and NHIS should strive to adhere to contractual agreements in terms of correct and timely claim reimbursement.

\section{Conclusions}

Through application of the Socio-anthropological model of Kleinman, our study demonstrates that important differences between EMs of clients, healthcare providers and NHIS exist and lead to differences in interpretation, expectations, and behavior. We made these differences explicit, validated and discussed the findings with the stakeholders. This Participatory Approach contributed to mutual learning and trust building and allowed jointly developing key messages and interventions to improve 'transactions' and trust building between clients, healthcare providers and the NHIS. Although our results cannot be extrapolated to other countries, we claim that Kleinman's model can help other scientists to explore service utilization problems.

\section{Acknowledgments}

This study was funded by the Netherlands Organization for Scientific Research, NWO-WOTRO. We thank all staff of the research team and all stakeholders who participated in this study.

\section{References}

[1] Carapinhaa, J. L., Ross-Degnanb, D., Destac, A, and Wagner, A. K. 2010. "Health Insurance Systems in Five Sub-Saharan African Countries: Medicine Benefits and Data for Decision Making." Health Policy doi:10.1016/j.healthpol.2010.11.009.

[2] Aryeetey, G., Jehu-Appiah, C., Spaan, E., Agyepong, I., and Baltussen, R. 2011. "Cost, Equity, Efficiency and Feasibility of Identifying the Poor in Ghana's National Health Insurance Scheme: Empirical Analysis of Various Strategies." Tropical Medicine and International Health 17 (1): 43-51.

[3] Lu, J. R., and Hsiao, W. C. 2003. "Does Universal Health Insurance make Health Care Unaffordable: Lessons from Taiwan." Health Affairs 22 (3): 77-88.

[4] Werned, W. 2009. "Micro-insurance in Bangladesh: Risk Protection for the Poor?" Journal of Health Population and Nutrition 27 (40): 563-73.

[5] Xu, K., Evans, D. B., Kawabata, K., Zeramdini, R., Klavus, J., and Murray, C. J. L. 2003. "Household Catastrophic Health Expenditures: a Multi-country Analysis." The Lancet 362: 111-7. 
[6] Asante, F., and Aikins, M. 2008. Does the NHIS cover the poor? Accra DANIDA working paper.

[7] Jehu-Appiah, C., Aryeetey, G., Agyepong, I., Spaan, E., and Baltussen, R. 2011. "Equity Aspects of the National Health Insurance Scheme in Ghana: Who is Enrolling, who is not and why?" Social Science \& Medicine 72: $157-65$.

[8] Osei-Akoto, I. 2003. "Demand for Voluntary Health Insurance by the Poor in Developing Countries: Evidence from Rural Ghana." CEA 37th Annual Meeting. Carleton University, Ottawa, Canada.

[9] Kamuzora, P., and Gilson, L. 2007. "Factors Influencing Implementation of the Community Health Fund in Tanzania." Health Policy and Planning 22: 95-102.

[10] Nketiah-Amponsah, E. 2009. "Demand for Health Insurance Among Women in Ghana: Cross Sectional Evidence." International Research Journal of Finance and Economics (33): 179-91.

[11] Mensah, J. 2010. "An Evaluation of the Ghana National Health Insurance Scheme in the Context of the Health MDG's." GDN Working Paper Seies. Washington, Global Development Network: 69.

[12] Sarpong, N., Loag, W., Fobil, J., Meyer, C. G., Adu Sarkodie, Y., May ,J., and Schwarz, N. G. 2010.

[13] Jutting, J. 2004. "Do Community-based Health Insurance Schemes Improve Poor People's Access to Health Care? Evidence from Rural Senegal." World Development 32 (2): 273-88.

[14] Vaughn, L. M., Jacquez, F., and Baker, R. C. 2009. "Cultural Health Attributions, Beliefs and Practise: Effects on Healthcare and Medical Education." The Open Medical Education Journal 2: 64-74.

[15] Horowitz, C., Rein, S., and Leventhal, H. 2004. "Patient Knowledge and Beliefs about the Care of Heart Failure: a Story of Maladies, Misconceptions and Mishaps." Social Science \& Medicine 58: 631-43.

[16] Dixon, J., Tenkorang, E. Y., and Luginaah, I. 2011. "Ghana's National Health Insurance Scheme: Helping the Poor or Leaving them behind?" Environment and Planning C: Government and Policy 29 (6): 1102-15.

[17] Schouten, B. C., and Meeuwesen, L. 2006. "Cultural Differences in Medical Communication: a Review of the Literature." Patient Education and Counseling 64: 21-34.

[18] Kleinman, A. 1978. "Concepts and Model for the Comparison of Medical Systems as Cultural Systems."
Social Science \& Medicine 12: 85-93.

[19] Kleinman, A. 2009. Kleinmans' Explanatory Models of Illness in Achieving Cultural.

[20] Helman, C. 2007. Culture Health and Illness. UK, Hodder Education Companion.

[21] Green, A. R., Carrilo, J., and Betancourt, J. R. 2002. Why the Disease-based Model of Medicine Fails.

[22] Adongo, P. B., Kirkwood, B., and Kendall, C. 2005. "How Local Community Knowledge about Malaria Affects Insecticide-treated Net Use in Northern Ghana." Tropical Medicine and International Health 10 (4): 366-78.

[23] Haidet, P., O'Malley, P., Kimberly, J., Barbara, F., Sharf, C., Alicia, P., Gladney, D., Greisinger, A., and Street Jr, R. 2008. "Characterizing Explanatory Models of Illness in Healthcare: Development and Validation of the CONNECT Instrument." Patient Education and Counseling 73: 232-9.

[24] Donfuet, H., Essombe, E., Mahieu, P., and Malin, E. 2011. "Social Capital and Willingness-to-pay for Community based Health Insurance in Rural Cameroon." Global Journal of Health Science 3 (1): 142-9.

[25] Fenenga, C. J., Nketiah-Amponsah, E., Bailey, A., and Hutter, I. 2015. "Participatory Action Approach for Client-centered Health Insurance." Action Research 0 (0): 1-19.

[26] Fenenga, C. J., Boahene, K., Arhinful, D. K., Rinke de Wit, T. F., and Hutter, I. 2014. "Do Prevailing Theories Sufficiently Explain Perceptions and Health Seeking Behavior of Ghanaians?" International Journal for Health Planning and Management 29 (1): 26-42.

[27] NHIA (2010, 2011). National health insurance Authority Annual report. Accra.

[28] Our Patients. West J. Medicine 176 (2): 141-3.

[29] Competency: A Case-Based Approach to Training Health Professionals. Edited by Hark, L., DeLisser, H.. Blackwell Publishing Ltd. ISBN: 978-1-405-18072-6.

[30] "National Health Insurance Coverage and Socio-economic Status in a Rural District of Ghana." Tropical Medicine and International Health 15 (2): 191-7.

[31] Zhang, L., Wang, H., Wang, L., and Hsiao, W. 2006. "Social Capital and Farmers Willingness -to-join a newly Established Community-based Health Insurance Scheme in Rural China." Health Policy 76: 233-42. 